\title{
L'aiélé (Canarium Schweinfurthii Engl.) : premier essai de plantation dans l'ouest du Cameroun
}

\author{
Raphaël Njoukama ${ }^{a}$ Régis Peltier ${ }^{b *}$
}

\author{
a Institut de recherche agricole \\ pour le développement (Irad), \\ BP 285, Foumban, \\ Cameroun \\ b Engref - Cirad-forêt, \\ BP 44494, \\ 34093 Montpellier Cedex 5, \\ France
}

* Correspondance et tirés à part

Reçu le 22 janvier 2002 Accepté le 18 mars 2002

Fruits, 2002, vol. 57, p. 239-248 (C) 2002 Cirad/EDP Sciences All rights reserved

DOI: $10.1051 /$ fruits:2002021

Resumen Español, p. 248

\section{Canarium Schweinfurthii: a first attempt at its domestication in the West of Cameroon.}

Abstract - Introduction. C. Schweinfurthii (Burseraceae) grows as isolated trees in the wet savanna of Cameroon. In the West of the country, the interest of the tree specially lies in the production of fruits, but C. Schweinfurthii is also exploited for its wood. Preliminary work for a more advanced domestication has been undertaken by Irad (Cameroon) since 1987. Materials and methods. The domestication work began with the development of a seed germination technique which finally made it possible to obtain an average rate of seedling emergence of $95 \%$ at the end of a 3 month period. Plantations then stayed in a tree nursery for 6 months before being transplanted to an experimental plot. Growth, flowering and fruit formation were then followed. Results. In the C. Schweinfurthii stand, the average survival rate decreased year after year and was stabilized at 84\% from 1992 to 1997 . It was only $42 \%$ in 2000 as a result of the damage caused by a bush fire in March 1998. In 2000, the average total height of the 12-year old trees reached $6.8 \mathrm{~m}$, and their trunks had a mean diameter of about $8.6 \mathrm{~cm}$. The dominant height and diameter were $10.8 \mathrm{~m}$ and $14.3 \mathrm{~cm}$, respectively. C. Schweinfurthii is a dioecious species. The formation of the first fruits happened with 8-year old trees. From 1996 to 2001, 19\% of the studied trees within the plot entered blooming or fruiting. Floral analyses showed that, during the same period, the experimental C. Schweinfurthii stand presented a sex ratio of $38 \%$ of male individuals for $62 \%$ of female trees. Conclusions. The preliminary work on the $C$. Schweinfurthiis domestication showed that the tree could produce after 8 years and that its growth in stands was satisfactory. Complementary studies will have to be undertaken. For example, the highlighting of vegetative differences between male and female seedlings at the nursery stage could allow an early sexing. Besides, the use of vegetative multiplication techniques could make it possible to give interested farmers only female trees, which are the only ones capable of fruiting.

Cameroon / Canarium Scbweinfurtbii / domestication / stand characteristics / population growth / flowering / fruiting / sex ratio / yields

\section{L'aiélé (Canarium Schweinfurthii Engl.) : premier essai de plantation dans l'ouest du Cameroun.}

Résumé - Introduction. L'aiélé (Burseraceae) pousse à l'état isolé en savane humide du Cameroun. Dans l'ouest du pays, l'intérêt de l'arbre réside surtout dans sa production de fruits, mais C. Schweinfurthii est aussi exploité pour son bois. En préalable à une domestication plus avancée, des travaux ont été entrepris par l'Irad (Cameroun) en 1987. Matériel et méthodes. Les travaux de domestication ont débuté par la mise au point d'une technique de germination des semences qui a finalement permis d'obtenir un taux moyen de levée de $95 \%$ au bout de 3 mois. Les plants ont ensuite séjourné en pépinière pendant 6 mois avant d'être transplantés en parcelle expérimentale. La croissance, la floraison et la production des arbres ont alors été suivies. Résultats. Le taux moyen de survie en plantation a peu à peu diminué de 1988 à 1992 pour se stabiliser à $84 \%$ de 1992 à 1997. Il était de $42 \%$ en 2000, par suite des dégâts occasionnés par un feu de brousse survenu en mars 1998. La hauteur totale moyenne des arbres à 12 ans a été de $6,8 \mathrm{~m}$ environ et leur diamètre moyen a été proche de $8,6 \mathrm{~cm}$. Les hauteur et diamètre dominants ont été respectivement de $10,8 \mathrm{~m}$ et 14,3 cm. L'aiélé est une espèce diö̈que. Les premières fructifications ont été observées sur des arbres âgés de 8 ans. De 1996 à 2001, $19 \%$ de l'effectif total de la parcelle sont entrés en floraison ou en fructification. Des analyses florales ont montré que, pendant la même période, le peuplement expérimental présentait une sex-ratio de $38 \%$ d'individus mâles pour $62 \%$ d'arbres femelles. Conclusions. Les travaux préliminaires de domestication de l'aiélé ont montré que l'arbre pouvait produire dès 8 ans et que sa croissance en peuplement était satisfaisante. Des études complémentaires devront être entreprises. Ainsi, la mise en évidence de différences végétatives entre plants mâles et femelles au stade pépinière pourrait permettre un sexage précoce. Par ailleurs, l'utilisation de techniques de multiplication végétative pourrait permettre de ne mettre à la disposition des paysans intéressés que des arbres femelles, seuls capables de fructifier.

Cameroun / Canarium Schweinfurtbii / domestication / caractéristique du peuplement / croissance de la population / floraison / fructification / sex ratio / rendement 


\section{Introduction}

L'aiélé ou " arbre aux fruits noirs" est une Burseracée qui pousse à l'état isolé en savane humide du Cameroun, notamment dans les différents systèmes agroforestiers. Dans cette région, l'arbre présente un port généralement trapu, un houppier dense et de grosses branches. Le port de forêt est plutôt élancé, avec un houppier peu dense. Le fût droit et cylindrique peut atteindre (25 à 40 ) $\mathrm{m}$ de haut et 1,50 $\mathrm{m}$ de diamètre. C'est une espèce fruitière forestière dont les multiples usages sont bien connus des populations rurales et même urbaines.

Dans l'Ouest-Cameroun, l'intérêt de l'arbre réside surtout dans sa production de fruits, mais C. Schweinfurthii est aussi exploité pour son bois. En effet, la pulpe charnue et résinifère du fruit est comestible après cuisson dans l'eau chaude à (40 à 50) ${ }^{\circ} \mathrm{C}$ pendant quelques dizaines de minutes. Elle accompagne les tubercules tels que le manioc, la patate douce et l'igname [1]. Les fruits font l'objet d'un commerce florissant. Tchouamo et al. signalent qu'en une saison de production, les fruits de l'aiélé ont rapporté aux seuls marchés des villages (figure 1) du département Mbamboutos une somme d'environ 27 millions de francs CFA [2]. En une saison, un arbre adulte, à houppier bien développé, peut produire

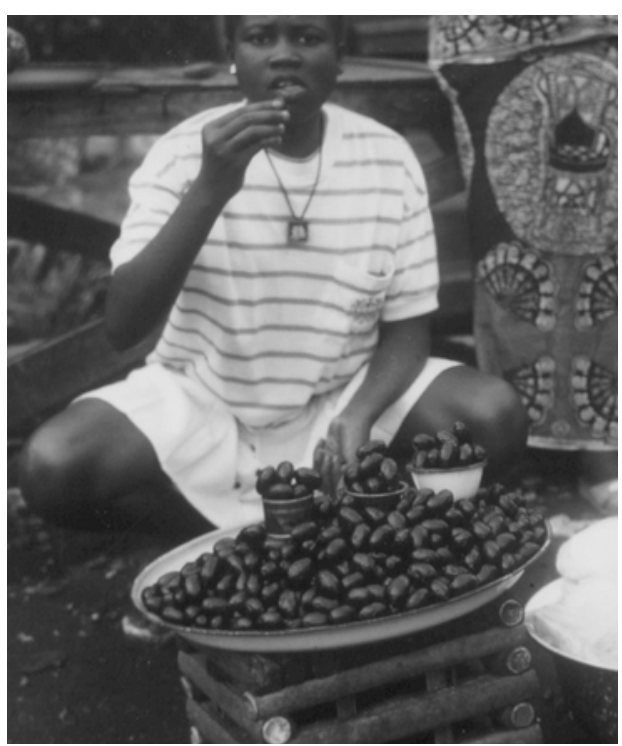

plus de $400 \mathrm{~kg}$ de fruits qui sont payés au producteur 25 FCFA par kg, soit un revenu de $15 €$ par arbre. Les autres usages du fruit ont une importance économique moindre. Cependant, au Nigeria, l'huile extraite de la pulpe a été testée avec satisfaction comme lubrifiant dans les moteurs Diesel [3]. Au sud-est de ce pays, les noyaux extraits des fruits sont utilisés pour fabriquer un collier qui, accroché à une calebasse constitue un instrument de musique [4]. Calcinés, ils rentrent dans le traitement de l'angine chez l'homme [5] et de la coccidiose chez la volaille [6].

Le deuxième intérêt économique de l'arbre dans l'Ouest-Cameroun vient de son bois utilisé en menuiserie légère. Il est recherché en sculpture et dans la fabrication d'ustensiles. Il entre également dans la fabrication des parquets, des huisseries et des caisses d'emballages [7]. L'écorce est l'un des ingrédients entrant dans la composition d'un philtre répandu dans les zones de forêt dense du Cameroun [8]. Une décoction de l'écorce s'emploie contre la colique [9], comme fortifiant chez les femmes enceintes et comme calmant en cas d'hémorroïdes [10].

La résine extraite par saignée de l'arbre sur pied sert à ravauder les calebasses fendues [11]. Séchée et broyée grossièrement, elle remplace efficacement l'encens importé qui est brûlé lors de grandes cérémonies par certaines confessions religieuses [12]. Elle est recherchée au Gabon pour ses propriétés émollientes, stimulantes et diurétiques [13].

Tous ces produits ligneux et non ligneux tirés de l'" arbre aux fruits noirs " sont exploités sous forme de "cueillette ". Il n'existe pratiquement pas de modes traditionnels de propagation de cette espèce pourtant bien appréciée en milieu paysan. La plupart des arbres présents dans les exploitations agricoles proviennent généralement de quelques plants sauvages survivants qui avaient été probablement protégés ou transplantés par le paysan à l'endroit de son choix.

En l'absence d'une véritable tradition de plantation de l'aiélé, des travaux ont été entrepris en 1987, en préalable à une 
domestication plus avancée. Ils ont permis d'étudier la croissance de l'arbre en conditions expérimentales et ses potentialités de production de fruits et de bois. Une attention particulière a été donnée à la mesure de la sex-ratio du peuplement, puisque, l'arbre étant diö̈que, seuls les plants femelles sont aptes à fructifier.

Les techniques de sylviculture choisies ont cherché à concilier la production de bois par plantation à forte densité au départ pour favoriser la formation d'un fût droit et sans branche d'au moins ( 5 ou 6) $\mathrm{m}$ et la production de fruits, par éclaircissage du peuplement dès identification possible des arbres femelles.

\section{Matériel et méthodes}

À maturité, les fruits de l'aiélé prennent une teinte violacée et renferment chacun un noyau épais, osseux et trigone [14] (figure 2). Les graines utilisées au cours de nos expérimentations ont été extraites de fruits récoltés sur des arbres isolés de la région de Foumban (province de l'Ouest-Cameroun).

\subsection{Test préalable de production des plants en pépinière}

Plusieurs techniques ont été testées pour hâter la germination des graines d'aiélé : eau chaude, eau bouillante, acide sulfurique concentré, etc. Le prétraitement qui a donné les meilleurs résultats a consisté en un dépulpage des "fruits noirs " après trempage dans de l'eau chaude à $(40-50){ }^{\circ} \mathrm{C}$. Ce même traitement qui ramollit la pulpe est d'ailleurs utilisé comme mode de cuisson des fruits avant leur consommation. Pour obtenir des semences d'aiélé, il a donc suffi de récupérer des noyaux issus de la consommation des fruits [5, 15].

Les graines ont été semées directement dans des sachets de polyéthylène remplis de terre fine humifère à raison d'une graine par sachet. Chaque graine a été déposée horizontalement dans le sachet et recouverte d'une mince couche de terre. À noter

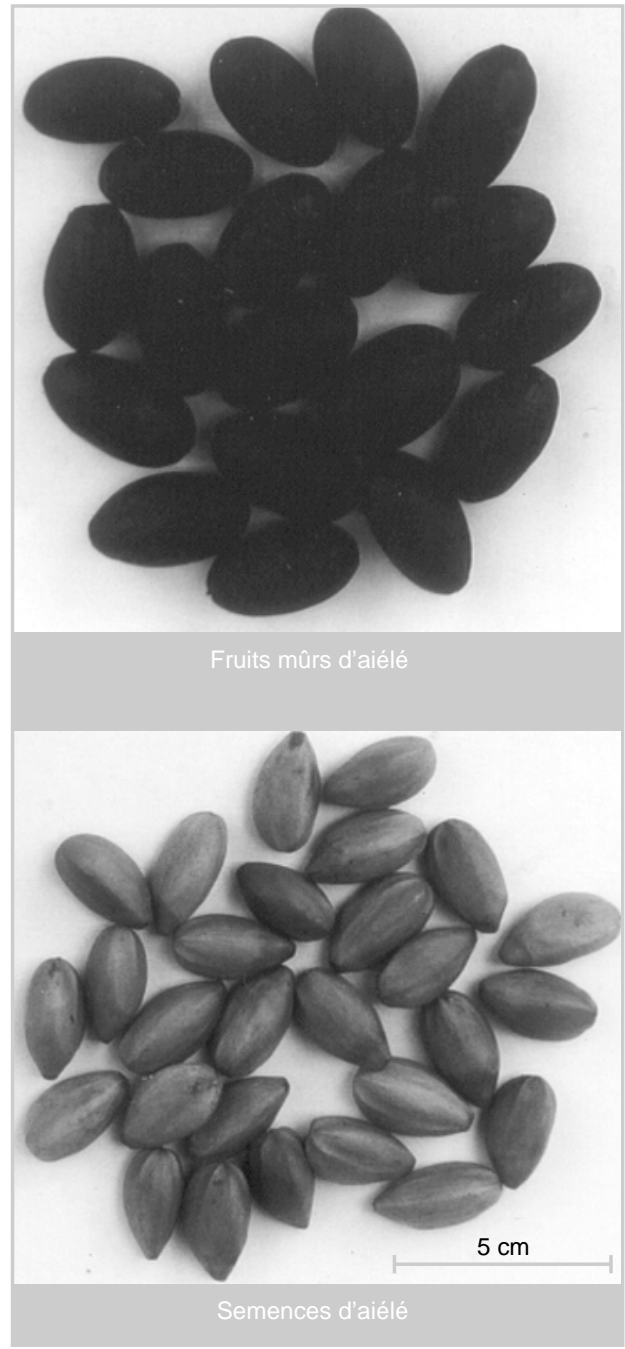

Figure 2.

Semences d'aiélé récupérées après ramollissement de la pulpe obtenu par cuisson des fruits [(40 à 50$\left.)^{\circ} \mathrm{C}\right]$. qu'un passage de ces graines en germoir suivi d'une opération de repiquage pourrait réduire la durée d'exposition aux intempéries de ces sachets dont les moins épais se sont révélés fragiles.

Les graines semées ont été copieusement arrosées quotidiennement. La technique de germination des semences ainsi mise au point a finalement permis d'obtenir un taux moyen de levée de $95 \%$ au bout de 3 mois

\subsection{Mise en place}

L'essai, d'une superficie totale de $2520 \mathrm{~m}^{2}$, a été installé en 1988 sur un sol alluvial de 
fond de vallée, relativement pauvre, de $\mathrm{pH}$ proche de 5, dans une parcelle de la réserve forestière de Melap, près de Foumban. La pluviométrie moyenne annuelle de la région est de $1900 \mathrm{~mm}$; elle se répartit de la mi-mars à la mi-novembre. La température moyenne annuelle y est de $21,4{ }^{\circ} \mathrm{C}$. C'est donc un climat tropical d'altitude comportant une saison sèche qui couvre la période de décembre à février.

Après un séjour de 6 mois en pépinière, les jeunes plants développés en sachet de polyéthylène ont été transplantés dans une parcelle expérimentale selon un dispositif simple constitué de quatre placeaux numérotés de 1 à 4 , de 120 plants chacun; le rythme de croissance de l'espèce en peuplement a ainsi pu être suivi. Sans a priori préalable sur le taux de survie envisageable, nous avons adopté une densité assez forte au départ correspondant à un écartement de $2 \mathrm{~m} \times 2 \mathrm{~m}$ tout en prévoyant d'effectuer des éclaircies ultérieures pour réduire progressivement cette densité.

Pendant les premières années, l'entretien des placeaux a été assuré par la pratique de cultures intercalaires (maïs, patates douces, etc.). Ensuite, le recrû a été rabattu manuellement, en fonction des besoins et de la disponibilité en personnel. Les placeaux contigus ont été presque homogènes, mais la végétation de l'un d'eux (le placeau 4) a cependant été plus vigoureuse.

\subsection{Mesures et observations d'ontogénie et de morphologie florale}

Le taux de survie des plants de l'ensemble de la parcelle a été évalué. Par ailleurs, pour apprécier la croissance des arbres en plantation, les mesures de hauteur et de circonférence à 1,30 m du sol ont été périodiquement effectuées. L'âge de la première fructification a également été déterminé par analyse florale. De même, l'aiélé étant une espèce dioïque, la sex-ratio des arbres ayant fleuri dans les placeaux expérimentaux a été mesurée.

\section{Résultats et discussions}

\subsection{Croissance en plantation}

Le taux moyen de survie des arbres de l'essai a diminué lentement au fil des ans et s'est stabilisé à 84 \% de mars 1992 à mars 1997. À la suite d'un feu de brousse qui a endommagé ces arbres en mars 1998, ce taux est tombé à $49 \%$ en septembre 1998 et avoisinait $42 \%$ en juin 2000 (tableau I).

Après 12 ans de croissance, la circonférence moyenne des arbres a été de $(26,9 \pm 10,5) \mathrm{cm}$, soit un diamètre moyen de $(8,6 \pm 3,3) \mathrm{cm}$ et la hauteur totale moyenne de $(6,8 \pm 2,5) \mathrm{m}$, soit un accroissement annuel moyen en hauteur de $0,57 \mathrm{~m}$ (figure 3). À noter que, pour ces mêmes arbres, la hauteur moyenne dominante a été de $(10,8 \pm 1,0) \mathrm{m}$ et la circonférence moyenne dominante de $(44,9 \pm 6,4) \mathrm{cm}$, correspondant à un diamètre moyen dominant de $(14,3 \pm 2,0) \mathrm{cm}$ (figure 4).

En juin 2000, les branches étaient bien développées et les houppiers évoluaient de plus en plus vers une canopée dense. L'éclaircie qui aurait dû être réalisée dans la parcelle a été volontairement différée afin de permettre la détermination de la sexratio des arbres.

Les courbes de la hauteur moyenne des aiélés, évaluée à l'échelle de chacun des placeaux, ont eu la même allure ; il en a été de même pour la circonférence moyenne des arbres. À noter cependant que, pour l'une et l'autre de ces caractéristiques, les arbres du placeau 1 sont apparus moins vigoureux (figure 4).

La distribution des troncs par classes de circonférence à 1,30 m du sol, réalisée pour les observations faites de 1998 à 2000, a été unimodale et caractéristique des peuplements constitués d'arbres de même âge ; elle s'est apparentée à une courbe de Gauss [16] (figure 5). En 1998, la distribution des tiges a montré une dissymétrie gauche traduisant le stade juvénile du peuplement alors âgé de 10 ans. Sa classe modale a été composée des individus de circonférence allant de (15 à 19) cm. À partir de 1999, cette classe modale s'est déplacée vers 
Tableau I.

Mesures de la croissance d'arbres de Canarium Schweinfurthii issus de la germination de graines et transplantés en 1988 en parcelle expérimentale constituée d'une parcelle de quatre placeaux de 120 plants chacun (OuestCameroun). Les nombres entre parenthèses correspondent aux écart-types.

\begin{tabular}{|c|c|c|c|c|c|c|c|c|c|c|c|}
\hline \multirow{2}{*}{$\begin{array}{l}N^{\circ} \text { du } \\
\text { placeau }\end{array}$} & \multicolumn{3}{|c|}{ Février 1990 (2 ans) } & \multicolumn{3}{|c|}{ Mars 1992 (4 ans) } & & & & & \\
\hline & $\begin{array}{c}\text { Taux de } \\
\text { survie } \\
(\%)\end{array}$ & \multicolumn{2}{|c|}{$\begin{array}{l}\text { Hauteur totale } \\
\text { moyenne } \\
(\mathrm{cm})\end{array}$} & $\begin{array}{l}\text { aux de } \\
\text { survie } \\
(\%)\end{array}$ & \multicolumn{2}{|c|}{$\begin{array}{l}\text { Hauteur totale } \\
\text { moyenne } \\
\text { (cm) }\end{array}$} & & & & & \\
\hline 1 & 94 & \multicolumn{2}{|c|}{$36,2(12,7)$} & 79 & \multicolumn{2}{|c|}{$108,8(38,1)$} & & & & & \\
\hline 2 & 93 & \multicolumn{2}{|c|}{$34,5(12,8)$} & 81 & \multicolumn{2}{|c|}{$109,0(36,0)$} & & & & & \\
\hline 3 & 96 & \multicolumn{2}{|c|}{$34,7(12,1)$} & 86 & \multicolumn{2}{|c|}{$104,2(39,6)$} & & & & & \\
\hline 4 & 98 & \multicolumn{2}{|c|}{$38,7(13,2)$} & 89 & \multicolumn{2}{|c|}{$127,5(44,6)$} & & & & & \\
\hline $\begin{array}{l}\text { Moyenne } \\
\text { générale }\end{array}$ & 95 & \multicolumn{2}{|c|}{$36,1(12,6)$} & 84 & \multicolumn{2}{|c|}{$112,7(40,6)$} & & & & & \\
\hline \multirow{2}{*}{$\begin{array}{l}\mathrm{N}^{\circ} \mathrm{du} \\
\text { placeau }\end{array}$} & \multicolumn{6}{|c|}{ Mars 1997 (9 ans) } & \multicolumn{5}{|c|}{ Septembre 1998 (10,5 ans) } \\
\hline & $\begin{array}{c}\text { Taux de } \\
\text { survie } \\
(\%)\end{array}$ & $\begin{array}{l}\text { Hauteur } \\
\text { totale } \\
\text { moyenne } \\
(\mathrm{m})\end{array}$ & $\begin{array}{c}\text { Hauteur } \\
\text { dominante } \\
\text { (m) }\end{array}$ & $\begin{array}{r}\text { Circon } \\
\text { dom }\end{array}$ & $\begin{array}{l}\text { :érence }{ }^{1} \\
\text { nante } \\
\text { m) }\end{array}$ & & $\begin{array}{c}\text { Taux de } \\
\text { survie } \\
(\%)\end{array}$ & $\begin{array}{l}\text { Hauteur } \\
\text { totale } \\
\text { moyenne } \\
(\mathrm{m})\end{array}$ & $\begin{array}{l}\text { Hauteur } \\
\text { dominante } \\
\text { (m) }\end{array}$ & $\begin{array}{l}\text { Circonférence }{ }^{1} \\
\text { moyenne } \\
(\mathrm{cm})\end{array}$ & $\begin{array}{c}\text { Circonférence } \\
\text { dominante } \\
(\mathrm{cm})\end{array}$ \\
\hline 1 & 79 & $3,6(1,7)$ & $8,0(1,1)$ & 29,6 & $(2,4)$ & & 40 & $5,0(2,2)$ & $8,5(1,3)$ & $20,3(7,9)$ & $35,0(2,0)$ \\
\hline 2 & 81 & $3,6(1,7)$ & $8,3(0,4)$ & 34,3 & $(3,5)$ & & 42 & $5,0(2,1)$ & $8,8(0,7)$ & $22,0(9,7)$ & $41,8(2,9)$ \\
\hline 3 & 84 & $3,9(1,7)$ & $8,7(0,4)$ & $33, \varepsilon$ & $(4,9)$ & & 50 & $5,6(2,1)$ & $9,1(0,7)$ & $22,6(8,3)$ & $37,4(4,5)$ \\
\hline 4 & 89 & $4,4(1,9)$ & $9,1(0,4)$ & 33, & $(2,5)$ & & 65 & $6,1(2,2)$ & $9,6(0,5)$ & $22,7(7,7)$ & $38,8(4,0)$ \\
\hline $\begin{array}{l}\text { Moyenne } \\
\text { générale }\end{array}$ & 84 & $3,9(1,8)$ & $8,5(0,8)$ & 32,8 & $(3,7)$ & & 49 & $5,5(2,2)$ & $9,0(0,9)$ & $22,0(8,3)$ & $38,5(4,1)$ \\
\hline \multirow{2}{*}{$\begin{array}{l}\mathrm{N}^{\circ} \mathrm{du} \\
\text { placeau }\end{array}$} & \multicolumn{6}{|c|}{ Septembre 1999 (11,5 ans) } & \multicolumn{5}{|c|}{ Juin 2000 (12 ans) } \\
\hline & $\begin{array}{l}\text { Taux de } \\
\text { survie } \\
(\%)\end{array}$ & $\begin{array}{l}\text { Hauteur } \\
\text { totale } \\
\text { moyenne } \\
(\mathrm{m})\end{array}$ & $\begin{array}{l}\text { Hauteur } \\
\text { dominante } \\
\text { (m) }\end{array}$ & $\begin{array}{r}\text { Circon } \\
\text { mo }\end{array}$ & $\begin{array}{l}\text { :érence }{ }^{1} \\
\text { enne } \\
\text { m) }\end{array}$ & $\begin{array}{l}\text { Circonférence }{ }^{1} \\
\text { dominante } \\
\text { (cm) }\end{array}$ & $\begin{array}{c}\text { Taux de } \\
\text { survie } \\
(\%)\end{array}$ & $\begin{array}{l}\text { Hauteur } \\
\text { totale } \\
\text { moyenne } \\
\text { (m) }\end{array}$ & $\begin{array}{l}\text { Hauteur } \\
\text { dominante } \\
\text { (m) }\end{array}$ & $\begin{array}{l}\text { Circonférence }{ }^{1} \\
\text { moyenne } \\
\text { (cm) }\end{array}$ & $\begin{array}{c}\text { Circonférence } \\
\text { dominante } \\
(\mathrm{cm})\end{array}$ \\
\hline 1 & 35 & $5,7(2,5)$ & $9,2(1,4)$ & 23,5 & $(9,9)$ & $36,6(3,4)$ & 34 & $6,1(2,6)$ & $10,8(1,4)$ & $25,0(10,9)$ & $40,6(3,9)$ \\
\hline 2 & 34 & $5,7(2,2)$ & $9,6(0,6)$ & 24,2 & $(10,5)$ & $43,2(6,6)$ & 33 & $6,1(2,5)$ & $10,4(0,7)$ & $26,25(11,9)$ & $47,4(7,3)$ \\
\hline 3 & 44 & $6,2(2,3)$ & $9,8(0,6)$ & 26,2 & $(8,8)$ & $41,0(6,1)$ & 43 & $6,9(2,4)$ & $10,5(0,4)$ & $27,8(10,4)$ & $45,0(6,3)$ \\
\hline 4 & 59 & $6,9(2,4)$ & $10,3(0,7)$ & 25, & $(8,5)$ & $43,0(6,4)$ & 57 & $7,6(2,5)$ & $11,4(1,1)$ & $27,8(9,5)$ & $46,4(7,4)$ \\
\hline $\begin{array}{l}\text { Moyenne } \\
\text { générale }\end{array}$ & 43 & $6,4(2,4)$ & $9,6(0,8)$ & 25 & $(9,3)$ & $41,0(5,9)$ & 42 & $6,8(2,5)$ & $10,8(1,0)$ & $26,9(10,5)$ & $44,9(6,4)$ \\
\hline
\end{tabular}

l'intervalle des circonférences de $(25$ à 29) $\mathrm{cm}$ et l'aplatissement un peu plus prononcé des distributions, qui s'est accentué en 2000, a confirmé l'accroissement du diamètre des arbres.

\subsection{Phénologie}

\subsubsection{Rythme foliaire}

En savane humide la défeuillaison et la feuillaison paraissent beaucoup plus 
Figure 3.

Plantation expérimentale de Canarium Schweinfurthii âgée de 12 ans.

Figure 4.

Évolution de la hauteur moyenne et de la circonférence moyenne des arbres de quatre placeaux (120 plants par placeau) d'un peuplement expérimental de Canarium Schweinfurthii.
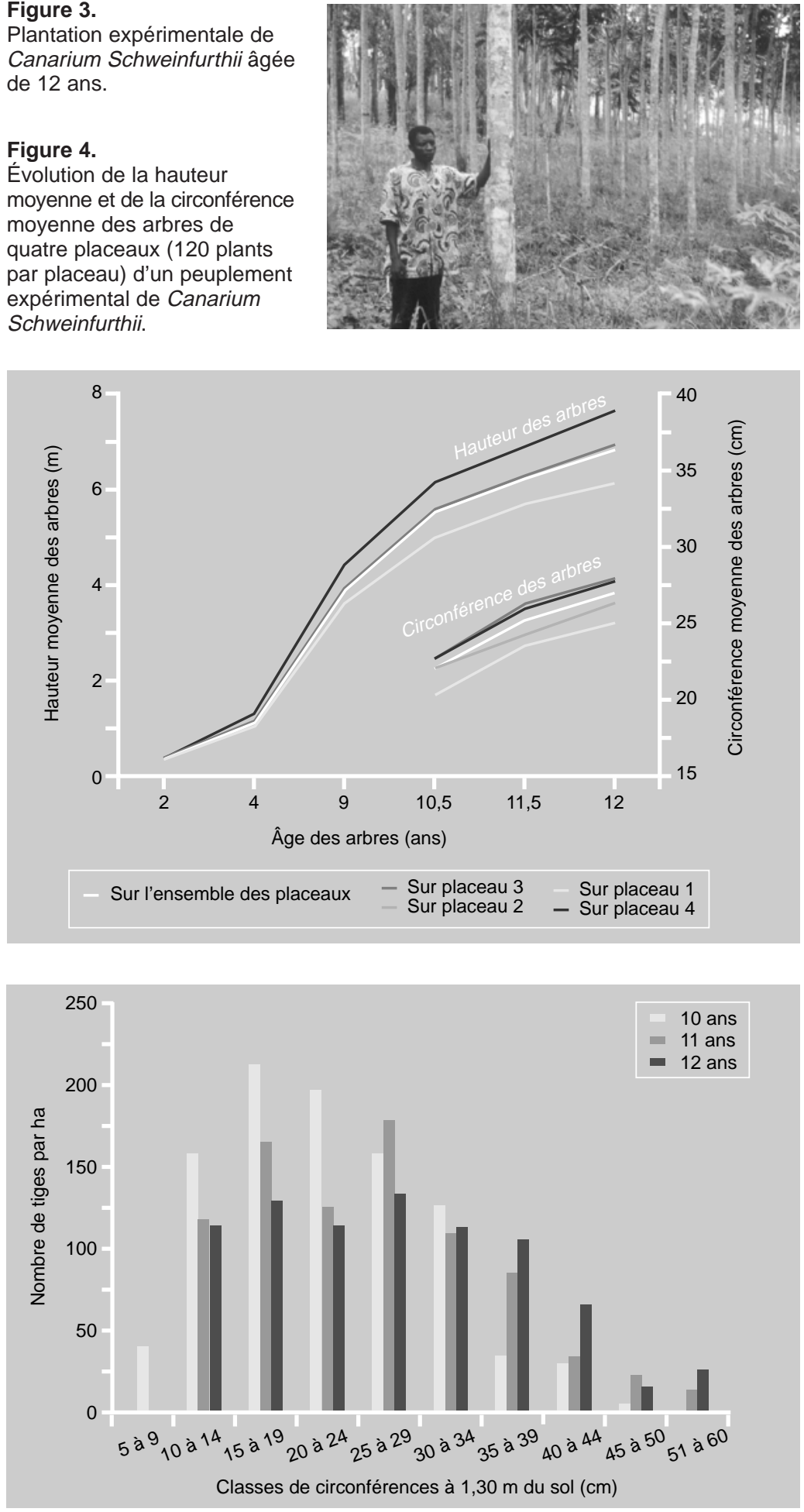

Figure 5.

Distributions d'arbres répartis en classes de circonférences mesurées à $1,30 \mathrm{~m}$ du sol pour des plants de Canarium Schweinfurthii âgés de (10 à 12) ans. régulières qu'en forêt dense. Elles se produisent généralement au début de la saison sèche, de novembre à janvier [17]. Le stade décidu de l'arbre (figure 6) peut durer jusqu'à 1 mois et coïncide généralement avec la période de maturation des fruits. Le caractère a été confirmé en plantation sans que des mesures précises ne soient effectuées.

\subsubsection{Floraison}

L'aiélé est une espèce diö̈que stricte. Les fleurs de $1 \mathrm{~cm}$ de long, groupées en inflorescences, se présentent en panicules à l'aisselle des feuilles terminales ; ces panicules, pubescentes à glabrescentes, atteignent (15 à 30) cm de long; leur couleur est blanc crème (figure 7). On distingue des fleurs mâles à six étamines et des fleurs femelles munies d'un ovaire à trois loges et d'un stigmate à trois lobes [18, 19]. L'ovaire est entouré de six staminodes.

Contrairement au rythme foliaire, la floraison est irrégulière. Elle se produit généralement après la feuillaison et peut être annuelle ou bisannuelle. Dans ce dernier cas, les deux vagues d'inflorescences ont été observées à (5 à 6) mois d'intervalle et cela aussi bien sur des arbres (mâles ou femelles) de la parcelle étudiée que sur des sujets isolés. La floraison bisannuelle, lorsqu'elle a lieu, pourrait expliquer la présence prolongée des fruits sur les marchés.

\subsubsection{Fructification}

Les individus mâles ne portent pas de fruits et les arbres femelles, même après floraison, ne fructifient pas automatiquement. Le fruit est une drupe ellipsoïdale (figure 8) qui devient violacée à maturité. La période qui s'étale entre la fructification et la maturation des fruits (stade des "fruits noirs ") est de (10 à 12) mois. Dans la parcelle étudiée, certains arbres ont donné leurs premiers fruits en 1996, à l'âge de 8 ans.

\subsection{Sex-ratio}

L'aiélé étant une espèce dioïque, il nous a paru intéressant de déterminer progressivement le pourcentage des mâles et celui des femelles dans le peuplement expérimental composé uniquement d'arbres francs de 
pied. Les premières observations ont d'abord permis d'identifier les arbres femelles à l'occasion de la première fructification des arbres plantés, qui a eu lieu en 1996. Ces observations se sont poursuivies les années suivantes, dès le stade de floraison. Nous avons pu ainsi procéder au sexage des arbres concernés à partir d'une analyse florale réalisée sur leurs fleurs.

De 1996 à 2001, 39 arbres (19 \% de la population) au total dans l'ensemble de la parcelle sont entrés en floraison ou en fructification (tableau II). Ils se sont répartis en $38 \%$ de pieds mâles et $62 \%$ d'individus femelles, soit une proportion d'un mâle pour moins de deux femelles. Cette supériorité en nombre des femelles sur les mâles, si elle s'accentuait par la suite, serait un indice encourageant pour les paysans intéressés par la production des fruits. Les observations se poursuivent actuellement et les prochaines analyses florales réalisées au fur et à mesure que d'autres arbres entrent en floraison permettront de déterminer la sex-ratio définitive des individus de la parcelle mise en place.

\section{Conclusion}

Une assertion paysanne de l'ouest-Cameroun dit que : "Celui qui plante l'aiélé ne mange pas toujours ses fruits ". Comme ceux-ci sont habituellement récoltés sur de très vieux arbres, les paysans ont tendance à croire que l'aiélé n'entre en fructification qu'à partir d'un âge très avancé. Dans la parcelle étudiée, les observations phénologiques ont prouvé le contraire en montrant que l'arbre pouvait produire ses premiers fruits vers l'âge de 8 ans.

L'assertion évoquée ci-dessus peut cependant se justifier dans le cas d'un paysan qui n'aurait planté qu'un ou deux sujets mâles. Ni lui, ni sa descendance ne pourraient alors récolter de fruits sur de tels arbres. En fait, la dioïcité de l'aiélé est un handicap pour sa propagation anthropique en milieu rural (figure 9) car, en repiquant un plant issu de graine, le paysan ne peut savoir s'il s'agit d'un pied mâle qui produira du bois mais jamais de fruits ou d'un pied

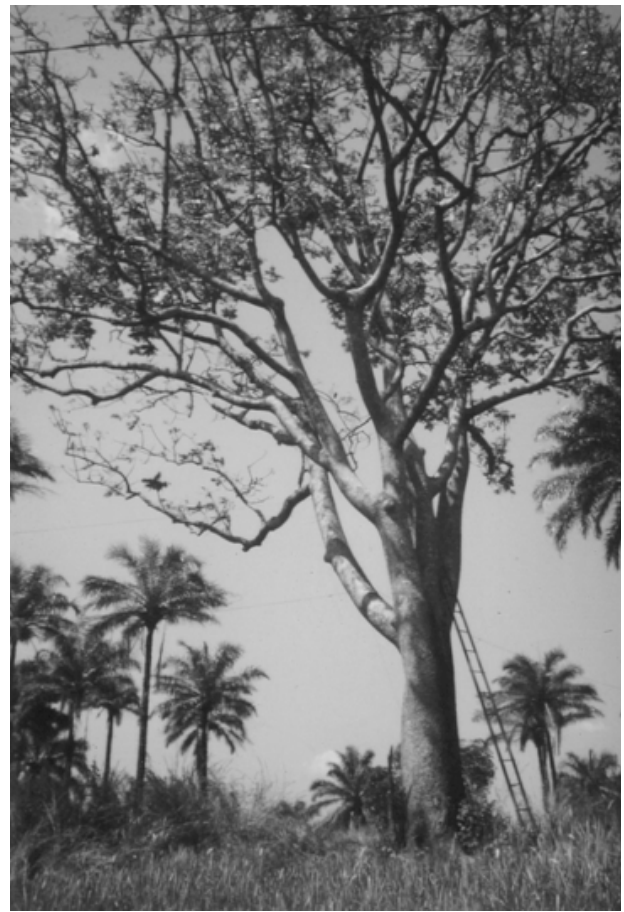

Figure 6.

Aspect de l'aiélé (Canarium Schweinfurthii Engl.) au stade décidu de l'arbre qui peut durer jusqu'à 1 mois et coïncide généralement avec la période de maturation des fruits.

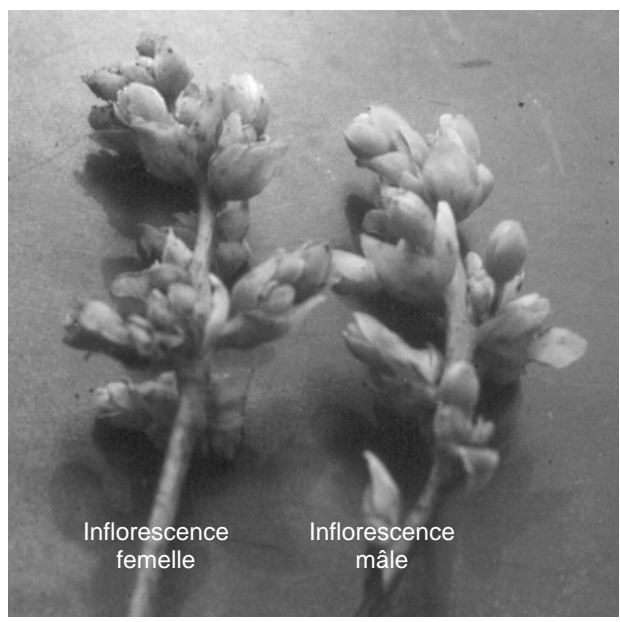

Figure 7.

Panicules d'un aiélé (Canarium Schweinfurthii Engl.) [(15 à 30) cm de long], de couleur blanc crème, formées d'inflorescences mâles ou femelles, l'espèce étant diöque stricte. femelle qui lui apportera un revenu plus précoce et plus régulier par sa production de fruits.

Si la sex-ratio définitive de la population d'arbres était connue, il serait possible d'estimer statistiquement la probabilité de planter un arbre femelle lors de la mise en place de plants issus de graines. Par ailleurs, l'utilisation de la macropropagation végétative pourrait permettre de pallier ce problème de plantation d'arbres femelles en ne mettant à 


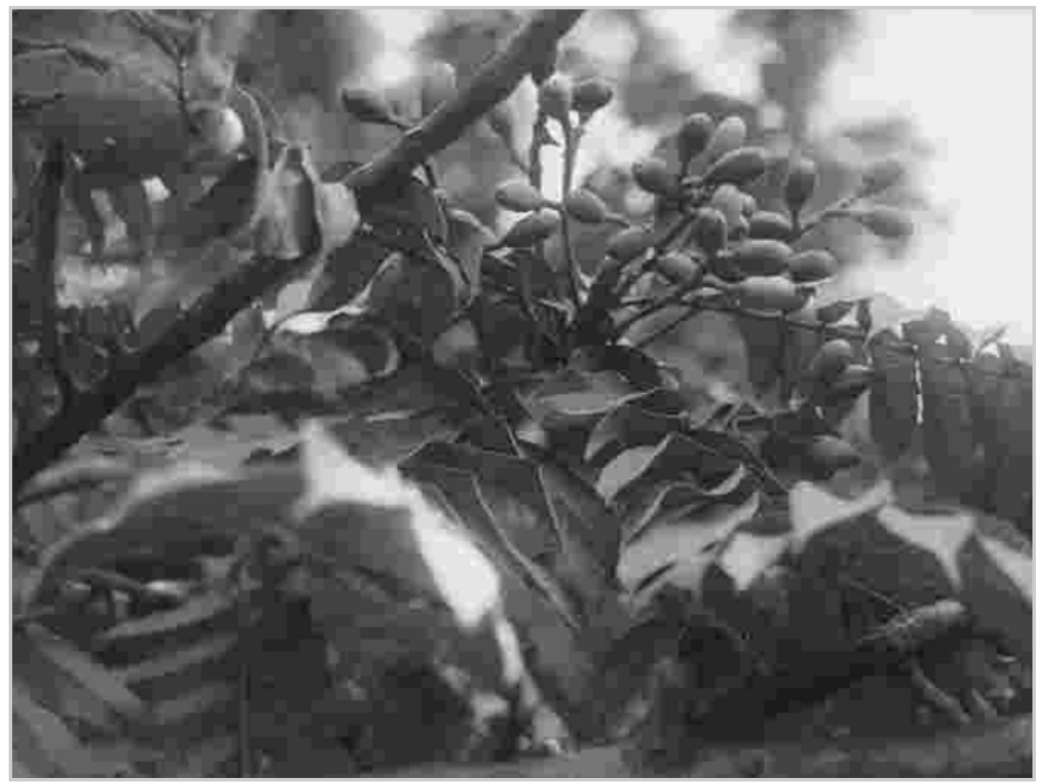

Figure 8.

Grappe de fruits d'aiélé (Canarium Schweinfurthii Engl.) de forme ellipsoïdale, en cours de maturation. la disposition des paysans intéressés que des arbres aptes à fructifier. Cette voie devra donc être explorée dans une prochaine étape de domestication par utilisation de toutes les techniques envisageables : greffage, marcottage aérien et bouturage. L'âge de la première fructification qui se situe actuellement à environ 8 ans pourrait aussi être réduit par utilisation d'une telle technique. Tant que la multiplication végétative ne pourra être effectuée, la mise en place d'un grand nombre de plants de C. Schweinfurthii est la seule technique qui peut être recommandée pour favoriser l'obtention d'un grand nombre de pieds femelles.

Sur le plan purement forestier, la croissance de l'aiélé en peuplement paraît satisfaisante. L'accroissement annuel en hauteur d'un arbre de 12 ans a été de $57 \mathrm{~cm}$ en moyenne. Il n'est certes pas comparable à celui d'essences exotiques comme Pinus Kesiya Royle dont l'accroissement annuel moyen atteindrait 1,28 m à 8 ans [20]. Mais, les arbres qui ont été plantés en parcelle expérimentale dans la région de Foumban sont vigoureux et de beaux fûts se dessinent progressivement. Une éclaircie s'avère pourtant nécessaire afin de favoriser la croissance future des troncs et par conséquent un bon développement du houppier qui sera nécessaire à une forte production de fruits.

De nombreux travaux de recherche devront encore être entrepris. Ainsi, une mise en évidence de différences végétatives entre plants mâles et femelles au stade pépinière pourrait permettre un sexage précoce. Par ailleurs, la production moyenne de fruits par arbre femelle et son évolution au fur et à mesure de la vie de l'arbre devra être évaluée. Enfin, différents taux d'éclaircie devraient être testés, afin de déterminer le nombre optimal d'arbres mâles à conserver dans le cas de l'exploitation de l'espèce pour la production de son bois.

Tableau II.

Sex-ratio mesurée de 1996 à 2001 pour un peuplement de Canarium Schweinfurthii issu de la transplantation en 1988 de plantules mises en parcelle expérimentale constituée de quatre placeaux de 120 plants chacun (Ouest-Cameroun).

$\begin{array}{cccccc}N^{\circ} \text { du placeau } & \begin{array}{c}\text { Effectif total } \\ \text { des arbres } \\ \left(E_{\text {total }}\right)\end{array} & \begin{array}{c}\text { Arbres en fleurs ou porteurs de fruits } \\ \text { \% par rapport à } \\ \left(E_{\text {total }}\right)\end{array} & \begin{array}{c}\text { \% mâles } \\ \text { par rapport à } \\ \left(E_{\text {fleurs/fruits }}\right)\end{array} & \begin{array}{c}\% \text { femelles } \\ \text { par rapport à } \\ \left(E_{\text {fleurs/fruits }}\right)\end{array} \\ 1 & 41 & 7 & 17 & 57 & 43 \\ 2 & 40 & 8 & 20 & 3 & 87 \\ 3 & 52 & 11 & 11 & 46 & 64 \\ 4 & 68 & 13 & 19 & 38 & 54 \\ \text { Ensemble des placeaux } & 201 & 39 & 19 & 38\end{array}$




\section{Références}

[1] Njoukam R., Germination des semences et croissance de l'Aiélé (Canarium Schweinfurthii Engl.) en plantation, in: Kapseu C., Kayem J. (Eds.), Actes du deuxième séminaire international sur la valorisation du safoutier et autres oléagineux non conventionnels, Ngaoundéré, Cameroun, décembre 1997, Presses Univ. Yaoundé, Cameroun, 1998, pp. 45-54.

[2] Tchouamo I.R., Tchoumboue J., Somonet M.A., Pinta J.Y., La commercialisation des fruits de l'aiélé (Canarium Schweinfurthii Engl.) dans les hautes terres de l'ouest du Cameroun, Riv. Ital. Sostanze Grasse LXXVII (2000) 680-686.

[3] Ajiwe V.I.E., Ajibla V., Martins C.M.O.A., Canarium Schweinfurthii oils: potential alternative source to diesel oil, in: Kapseu C., Kayem J., Actes du séminaire international sur le séchage et la valorisation du karité et de l'aiélé, Ngaoundéré, Cameroun, décembre 1999, Presses Univ. Yaoundé, Cameroun, 2000, pp. 145-155.

[4] Burkill H.M., The useful plants of west tropical Africa, Ed. 2, Vol. 1, Families A-D, Royal Botanic Gardens, Kew, UK, 1985, 960 p.

[5] Njoukam R., L'arbre aux fruits noirs : l'aiélé, Le Flamboyant 46 (1998) 11-15.

[6] Tchoumboue J., Meutchieye F., Mpoame M., Utilisation du noyau du fruit de Canarium Schweinfurthii Engl. dans le traitement de la coccidiose aviaire, in: Kapseu C., Kayem J., Actes du séminaire international sur le séchage et la valorisation du karité et de l'aiélé, Ngaoundéré, Cameroun, décembre 1999, Presses Univ. Yaoundé, Cameroun, 2000, pp. 125-133.

[7] Fortin Y., Poliquin J., Durabilité naturelle et préservation de cent bois tropicaux africains, Cent. Rech. Dév. Int., IDRC-017 f, 1974, $143 \mathrm{p}$.

[8] Tchouamo I.R., Tchoumboue J., Pinta J.Y., Njoukam R., L'aiélé (Canarium Schweinfurthii Engl.) : plante oléagineuse à usages multiples en Afrique, Riv. Ital. Sostanze Grasse LXXVII (2000) 677-680.

[9] Tchouamo I.R., Njoukam R., Étude de quelques ligneux utilisés en médecine traditionnelle par les Bamiléké des hauts plateaux de l'ouest du Cameroun, Ethnopharmacologia 26 (2000) 14-22.

[10] Bouquet A., Debray M., Plantes médicinales de la Côte-d'Ivoire, Travaux et documents de l'Orstom (Paris) 32, Paris, France, 1974, 232 p.

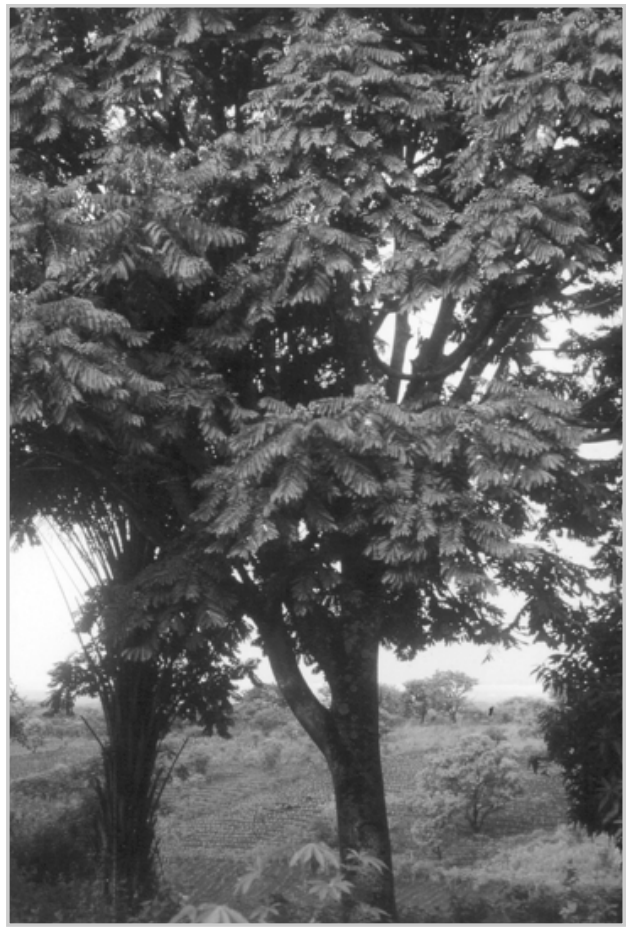

Figure 9.

Aiélé (Canarium Schweinfurthii Engl.) adulte propagé par l'homme en milieu rural.
[11] Gautier D., Les principales espèces ligneuses de Bafou (Ouest-Cameroun) et leurs utilisations, Inader-Cuds / Engref, Gret, Montpellier, France, 1991, $198 \mathrm{p}$.

[12] Njoukam R., Croissance, développement et usages de l'aiélé (Canarium Schweinfurthii Engl.) en savane humide du Cameroun, in: Kapseu C., Kayem J., Actes du séminaire international sur le séchage et la valorisation du karité et de l'aiélé, Ngaoundéré, Cameroun, décembre 1999, 2000, pp. 89-103.

[13] Raponda-Walker A., Sillans R., Les plantes utiles du Gabon, Édition Paul Lechevalier, Paris, 1961, $614 \mathrm{p}$.

[14] Anonyme, Recueil de fiches techniques : aiélé, Revue BFT (CTFT, Nogent-sur-Marne) 187 (1979) 65-78.

[15] Njoukam R., Effets de différents prétraitements sur la germination des semences de l'aiélé (Canarium Schweinfurthii Engl.), Rev. Sci. Tech. Sér. Agron. Zootech. (Minrest/Irad nº spécial 1987 1997) 3 (4) (1997) 67-81.

[16] Rondeux J., La mesure des arbres et des peuplements forestiers, Presses Agron. Gembloux, Belgique, 1993, 521 p.

[17] Ndankep Tchakounte H., Monographie de l'aiélé (Canarium Schweinfurthii Engl.), Mém. Ensa-Cuds, Dschang, Cameroun, 1988, 84 p. 
[18] Vivien J., Faure J.J., Arbres des forêts denses d'Afrique centrale, République unie du Cameroun / Ministère français des relations extérieures, de la coopération et du développement / CTFT, Paris, France, 1986, 565 p.
[19] Vivien J., Faure J.J., Fruitiers sauvages du Cameroun. Burséracées, Fruits 43 (9) (1988) 509.

[20] Njoukam R., Section de recherches forestières en savane d'altitude, Ira CRF, Rapp. Tech. 1987, Foubam, Cameroun, 1988, 46 p.

\section{El aiele (Canarium Schweinfurthii Engl.) primer ensayo de plantación en el oeste de Camerún.}

Resumen - Introducción. El aiele es una burserácea que crece de forma aislada en la sabana húmeda de Camerún. En el oeste del país, el interés del árbol reside sobre todo en su producción de fruta, pero también se explota C. Schweinfurthii por su madera. El Irad (Camerún) emprendió una serie de trabajos en 1987 como paso previo a una domesticación más avanzada. Material y métodos. Los trabajos de domesticación comenzaron con la puesta a punto de una técnica de germinación de las semillas que, finalmente, permitió obtener un porcentaje medio de emergencia del $95 \%$ al cabo de 3 meses. A continuación, las plantas se colocaron en vivero durante 6 meses antes de trasplantarse en parcela experimental. Se efectuó entonces un seguimiento del crecimiento, floración y producción de los árboles. Resultados. El porcentaje promedio de supervivencia en plantación disminuyó poco a poco de 1988 a 1992 para estabilizarse en el 84\% de 1992 a 1997. Era del 42\% en 2000, a consecuencia de los daños causados por un fuego de matorral ocurrido en marzo de 1998. La altura total media de los árboles a los 12 años era de $6,8 \mathrm{~m}$ aproximadamente y su diámetro promedio cercano a $8,6 \mathrm{~cm}$. La altura y diámetro dominantes fueron respectivamente de $10,8 \mathrm{~m}$ y $14,3 \mathrm{~cm}$. El aiele es una especie dioica. Las primeras fructificaciones se observaron en árboles de 8 años. De 1996 a 2001 el 19\% del conjunto total de la parcela entró en floración o en fructificación. Los análisis florales mostraron que, durante el mismo período, el rodal experimental presentaba una razón de sexos del 38\% de individuos masculinos por un $62 \%$ de árboles femeninos. Conclusiones. Los trabajos preliminares de domesticación del aiele pusieron de manifiesto que el árbol podía producir a partir de 8 años y que su crecimiento en rodal era satisfactorio. Deberán acometerse estudios complementarios. De este modo, la puesta en evidencia de diferencias vegetativas entre plantas masculinas y femeninas en la fase de vivero podría permitir un sexaje precoz. Por otra parte, la utilización de técnicas de multiplicación vegetativa podría permitir poner a disposición de los campesinos interesados árboles femeninos, los únicos capaces de fructificar.

Camerún / Canarium Schweinfurtbii / domesticación / características del rodal / crecimiento de la población / floración / fructificación / proporción de los sexos / rendimiento

To access this journal online:

www.edpsciences.org 